\title{
Hydrothermal Synthesis of Polyhedral Nickel Sulfide by Dual Sulfur Source for Highly-Efficient Hydrogen Evolution Catalysis
}

\author{
Yuan Gao ${ }^{1}$, Ka Wang ${ }^{1}$, Zixia Lin ${ }^{2}$, Haizeng Song ${ }^{3}$, Xiaomeng Duan ${ }^{4}$, Zehui Peng ${ }^{1}$ and \\ Shancheng Yan ${ }^{1, *(\mathbb{D})}$ \\ 1 School of Geography and Biological Information, Nanjing University of Posts and Telecommunications, \\ Nanjing 210023, China; 1218063835@njupt.edu.cn (Y.G.); 1217063729@njupt.edu.cn (K.W.); \\ 1019172222@njupt.edu.cn (Z.P.) \\ 2 Testing Center, Yangzhou University, Yangzhou 225009, China; zxlin@yzu.edu.cn \\ 3 School of Electronic Science and Engineering, Nanjing University, Nanjing 210093, China; \\ dg1823028@smail.nju.edu.cn \\ 4 Jiangsu National Synergetic Innovation Center for Advanced Materials (SICAM), \\ Institute of Advanced Materials (IAM), Nanjing University of Posts \& Telecommunications, \\ Nanjing 210023, China; 1218063822@njupt.edu.cn \\ * Correspondence: yansc@njupt.edu.cn
}

Received: 4 October 2020; Accepted: 21 October 2020; Published: 24 October 2020

check for updates

\begin{abstract}
Transition metal sulfides are cheap and efficient catalysts for water splitting to produce hydrogen; these compounds have attracted wide attention. Nickel sulfide $\left(\mathrm{NiS}_{2}\right)$ has been studied in depth because of its simple preparation process, excellent performance and good stability. Here, we propose a modification to the hydrothermal synthesis method for the fabrication of a highly efficient and stable $\mathrm{NiS}_{2}$ electrocatalyst prepared by two different sulfur sources, i.e., sulfur powder and $\mathrm{C}_{3} \mathrm{H}_{7} \mathrm{NaO}_{3} \mathrm{~S}_{2}$ (MPS), for application in hydrogen evolution reactions. The obtained $\mathrm{NiS}_{2}$ demonstrated excellent HER performance with an overpotential of $131 \mathrm{mV}$ to drive $-10 \mathrm{~mA} \mathrm{~cm}^{-1}$ in $0.5 \mathrm{M} \mathrm{H}_{2} \mathrm{SO}_{4}$ solution with $5 \mathrm{mV}$ performance change after 1000 cycles of stability testing. We believe that this discovery will promote the industrial development of nonprecious metal catalysts.
\end{abstract}

Keywords: transition metal sulfide; hydrogen evolution reaction; electrocatalyst

\section{Introduction}

Fossil fuels have been extremely important over the past hundred years, at the same time causing very serious damage to the environment [1-3]. In order to alleviate the pressure on the ecological environment, people have begun to look for more green, efficient and low-cost methods of energy generation [4-6]. Hydrogen is a clean energy source; it has a calorific value higher than fossil fuels and may be produced from water without pollution to the environment [7]. Therefore, the usage of catalysts to optimize the decomposition of water for hydrogen production has attracted great attention from researchers [8-12]. At the present, there are a variety of extremely stable and high-efficiency catalysts, such as platinum and palladium; however, their application is limited by their high cost $[13,14]$. Thus, there is a need to find a low-cost and stable catalyst.

Nonprecious transition metals are abundant and exhibit a good performance for hydrogen evolution reactions [15]. Nonprecious transition metal derivatives such as oxides, sulfides, carbides, nitrides, and alloys have been studied, and all demonstrated good catalytic performance. As a transition metal, nickel-based materials have been thoroughly studied in terms of catalysis [1,16-19]. Nickel-based dichalcogenides, specifically, $\mathrm{NiS}_{2}$, are narrow-bandwidth semiconductors performing 
well in acidic environments with a relatively small Tafel slope and a low overpotential [17,20-23]. Some $\mathrm{NiS}_{2}$ crystals have irregular morphologies and complicated structures, causing instability in the performance of the catalyst. If there is further improvement in the catalytic performance and stability of $\mathrm{NiS}_{2}$, it is expected to be widely used in industry.

In this work, we propose a hydrothermal synthesis method of polyhedral $\mathrm{NiS}_{2}$ (MS) from $\mathrm{C}_{3} \mathrm{H}_{7} \mathrm{O}_{3} \mathrm{NaS}_{2}$ (MPS) and sulfur powder used as the dual sulfur source. The obtained $\mathrm{NiS}_{2}$ was used for hydrogen evolution reactions (HER), performed in an acidic environment of $0.5 \mathrm{M} \mathrm{H}_{2} \mathrm{SO}_{4}$. The catalyst showed excellent performance: the turn-on voltage at a current density of $10 \mathrm{~mA} \mathrm{~cm}{ }^{-2}$ was $131 \mathrm{mV}$, and the Tafel slope was $50 \mathrm{mV} \mathrm{dec}^{-1}$. In order to ensure the relevance of this technique for practical applications, we also conducted a stability test of 1000 circles; the resulting performance change was only $5 \mathrm{mV}$. For the renewable energy industry, we believe that this method of preparation of $\mathrm{NiS}_{2}$ electrodes will promote the development of electrocatalysts.

\section{Materials and Methods}

\subsection{Materials and Chemicals}

The carbon fiber paper (CFP) model was obtained from CeTech Co., Ltd. (WOS1009) (Taichung City, Taiwan). $\mathrm{Ni}\left(\mathrm{NO}_{3}\right)_{2} \cdot 6 \mathrm{H}_{2} \mathrm{O}$ was obtained from Shanghai Titan Technology Co., Ltd. (Shanghai, China). Sulfur powder (S) was obtained from Nanjing Chemical Reagent Co., Ltd. (Nanjing, China). Sodium 3-mercapto-1-propanesulfonate $\left(\mathrm{C}_{3} \mathrm{H}_{7} \mathrm{NaO}_{3} \mathrm{~S}_{2}, \mathrm{Na}\left[\mathrm{SH}\left(\mathrm{CH}_{2}\right)_{3} \mathrm{SO}_{3}\right], \mathrm{MPS}\right)$ was obtained from Aladdin Reagent Co., Ltd. (Shanghai, China). A deionized water Millipore filter was obtained from Millipore Q, USA (Millipore Q, Billerica, MA, USA).

\subsection{Synthesis of $\mathrm{NiS}_{2}$ (MS) with Dual Sulfur Source}

Cleaned carbon fiber paper (CFP), washed using deionized water and absolute ethanol, was prepared. Then, $1.2 \mathrm{mM}$ of $\mathrm{Ni}\left(\mathrm{NO}_{3}\right)_{2} \cdot 6 \mathrm{H}_{2} \mathrm{O}$ and $1.6 \mathrm{mM}$ of MPS were mixed into the Teflon-lined autoclave with $25 \mathrm{~mL}$ deionized water and stirred for $15 \mathrm{~min}$. After the above step, $0.8 \mathrm{mM}$ of sulfur powder was added to the solution and stirred for 15 min slowly. After finishing this step, the CFP was placed into a container and was heated to $180^{\circ} \mathrm{C}$ for $8 \mathrm{~h}$. After this, the CFP was taken out and washed.

\subsection{Synthesis of $\mathrm{NiS}_{2}$ with MPS}

Cleaned carbon fiber paper (CFP), washed by deionized water and absolute ethanol, was prepared. Then, $1.2 \mathrm{mM}$ of $\mathrm{Ni}\left(\mathrm{NO}_{3}\right)_{2} \cdot 6 \mathrm{H}_{2} \mathrm{O}$ and $1.6 \mathrm{mM}$ of MPS were added to the Teflon-lined autoclave with $25 \mathrm{~mL}$ deionized water and stirred for $15 \mathrm{~min}$. After finishing this step, the CFP was placed into a container and heated to $180^{\circ} \mathrm{C}$ for $8 \mathrm{~h}$. After this, the CFP was taken out and washed.

\subsection{Synthesis of $\mathrm{NiS}_{2}$ with $\mathrm{S}$}

Cleaned carbon fiber paper (CFP), washed by deionized water and absolute ethanol, was prepared. Then, $1.2 \mathrm{mM}$ of $\mathrm{Ni}\left(\mathrm{NO}_{3}\right)_{2} \cdot 6 \mathrm{H}_{2} \mathrm{O}$ was added to the Teflon-lined autoclave with $25 \mathrm{~mL}$ deionized water and stirred for $15 \mathrm{~min}$. After the above step, $0.8 \mathrm{mM}$ of sulfur powder was added to the solution and stirred for $15 \mathrm{~min}$ slowly. After finishing this step, put CFP was placed into a container and heated to $180^{\circ} \mathrm{C}$ for $8 \mathrm{~h}$. Finally, the CFP was taken out and washed.

\subsection{Materials Characterization}

X-ray diffractometer (XRD) by Bruker D8 Advance X-ray diffractometer (XRD) with Cu-K $\alpha$ radiation $\left(15^{\circ}\right.$ to $75^{\circ}, 0.1^{\circ} \mathrm{s}^{-1}$ ) (Bruker Daltonics Inc., Karlsruhe, Germany). Raman measurements by Horiba LabRAM system (HORIBA, Ltd., Kyoto, Japan). SEM images by Scanning electron microscopy (FE-SEM; JSM-7000F JEOL Ltd., Tokyo, Japan). TEM and HRTEM images by JEOL type JEM2100 instrument (JEOL Ltd., Tokyo, Japan). XPS was performed on as-synthesized $\mathrm{NiS}_{2}$ by PHI5000 Versaprobe (Ulvac-Phi Inc., Kanagawa, Japan). 


\subsection{Electrochemical Measurements}

A CHI760E electrochemical analyzer (CH Instruments, Chenhua Co., Shanghai, China) was used to analyze the performance of samples in $0.5 \mathrm{M} \mathrm{H}_{2} \mathrm{SO}_{4}$. A three-electrode system was used to test samples. The measured potentials were converted to a reversible hydrogen electrode $\left(\mathrm{E}(\mathrm{RHE})=\mathrm{E} \mathrm{Hg} / \mathrm{Hg}_{2} \mathrm{Cl}_{2}+0.241+0.0591 \mathrm{pH}\right)$. Prior to testing, nitrogen had to be bubbled into $\mathrm{H}_{2} \mathrm{SO}_{4}$ solution to remove oxygen from the solution. The range of LSV test was from -0.8 to $0 \mathrm{~V}$ with the scan rate of $2 \mathrm{mV} \mathrm{s}^{-1}$. Tafel data could be calculated from the LSV test. Different scan rates of the CV test ( 5 to $90 \mathrm{mV} \mathrm{s}^{-1}$ ) were carried out to reveal the ECSA of samples. EIS measurements were carried out in a frequency range from $10^{5}$ to $0.1 \mathrm{~Hz}$ with an AC voltage of $5 \mathrm{mV}$.

\section{Results and Discussion}

Figure 1a,b present scanning electron microscopy (SEM) images of $\mathrm{NiS}_{2}$ (MS) prepared by sulfur powder and MPS, forming a regular polyhedral block structure on the carbon fiber which was from the carbon fiber paper (CFP). Transmission electron microscopy image (TEM) captured from the edge of the sample is shown on Figure 1c. Figure 1d represents the HRTEM image of $\mathrm{NiS}_{2}(\mathrm{MS})$, with regular crystal plane spacing of $0.283 \mathrm{~nm}$, corresponding to the (200) plane according to the following XRD card (JCPDS\#65-3325) [24,25]. In order to compare the proposed $\mathrm{NiS}_{2}$ (MS) synthesis method, i.e., from dual sulfur sources, with other techniques, we also studied the morphology (Figures S1 and S2) of the synthesized material by two other techniques. As can be seen from Figure S1a,b, the carbon fiber surface also has some regularly shaped products stacked together. However, only a small amount of product adhered to the surface compared with the material synthesized by the dual sulfur source, which had a certain degree of negative influence on the conductivity between the substances. Figure S2 is SEM patterns of $\mathrm{NiS}_{2}$ synthesized by sulfur powder as the sole sulfur source. The resulting product was relatively small and scattered on the surface of the carbon fiber, which was in sharp contrast to the products synthesized by the previous two schemes. During the HER process, the other two samples easily detached from the carbon fiber, resulting in poor performance and stability. In comparison, the samples prepared by the dual sulfur source interlaced with each other provided a larger coverage of the electrode, and thus, improved resistance and catalytic performance.
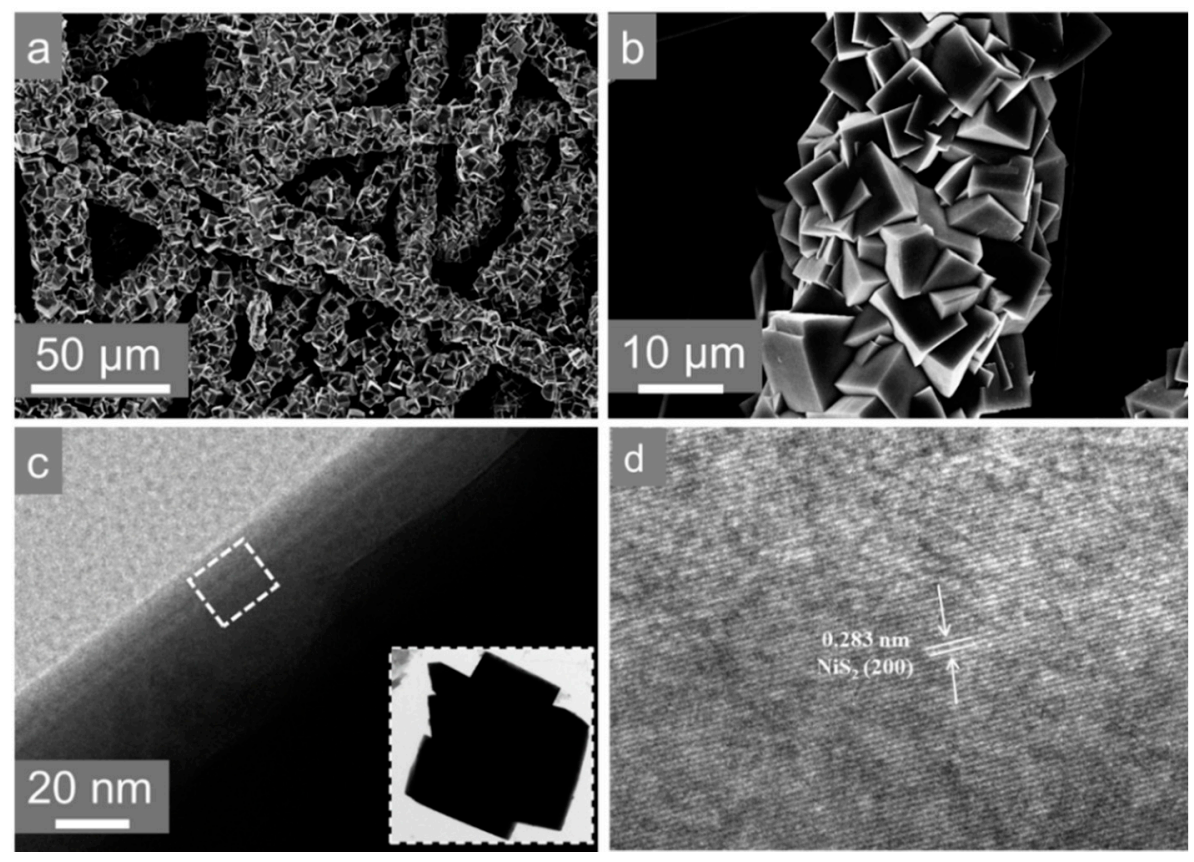

Figure 1. (a,b) Scanning electron microscopy images of $\mathrm{NiS}_{2}$; (c) TEM image and (d) HRTEM image of $\mathrm{NiS}_{2}$. 
X-ray diffraction (XRD) analysis was performed in order to investigate the structure and composition of the obtained $\mathrm{NiS}_{2}$ [25]. Analyzing the shape of the peaks in the XRD picture, it can be seen that the obtained substance had good crystallinity. The diffraction peaks of $31.7^{\circ}, 36.7^{\circ}$, $41.3^{\circ}, 45.4^{\circ}, 52.9^{\circ}, 56.4^{\circ}$ and $62.9^{\circ}$ corresponded to $\mathrm{NiS}_{2}$ planes according to JCPDS\#65-3325 of (111), (200), (210), (211), (220), (221) and (311), respectively. The diffraction peak at $27^{\circ}$ was attributed to the presence of carbon fiber [24]. The peak at $65^{\circ}$ may be have been contributed to the presence of sulfur. Figure $2 b$ represents the full $x$-ray photoelectron spectroscopy (XPS) spectra of $\mathrm{NiS}_{2}$ (MS) used for confirmation of its elemental composition $[17,26]$. As shown in Figure 2c, two spin-orbit doublets and two satellites can be deconvoluted from the Ni $2 p$ spectrum, namely $853.5 \mathrm{eV}$ and 856.1 $\mathrm{eV}$ corresponded to $2 \mathrm{p} 3 / 2$ of $\mathrm{Ni}^{2+}$ and $\mathrm{Ni}^{3+}$, and $871.1 \mathrm{eV}$ and $874.5 \mathrm{eV}$ were attributed to $2 \mathrm{p} 1 / 2$ of $\mathrm{Ni}^{2+}$ and $\mathrm{Ni}^{3+}$ respectively $[17,26-29]$. Figure $2 \mathrm{~d}$ shows the deconvolution of $\mathrm{S} 2 \mathrm{p}$ peaks: the peak at $161.52 \mathrm{eV}$ belonged to $\mathrm{S} 2 \mathrm{p} 3 / 2$, while that at $162.87 \mathrm{eV}$ corresponded to $\mathrm{S} 2 \mathrm{p} 1 / 2$ of Ni-S bondings [30,31]. Due to the surface oxidation of $\mathrm{NiS}_{2}$, the peak at $166.7 \mathrm{eV}$ could be attributed to S-O bonding [19].
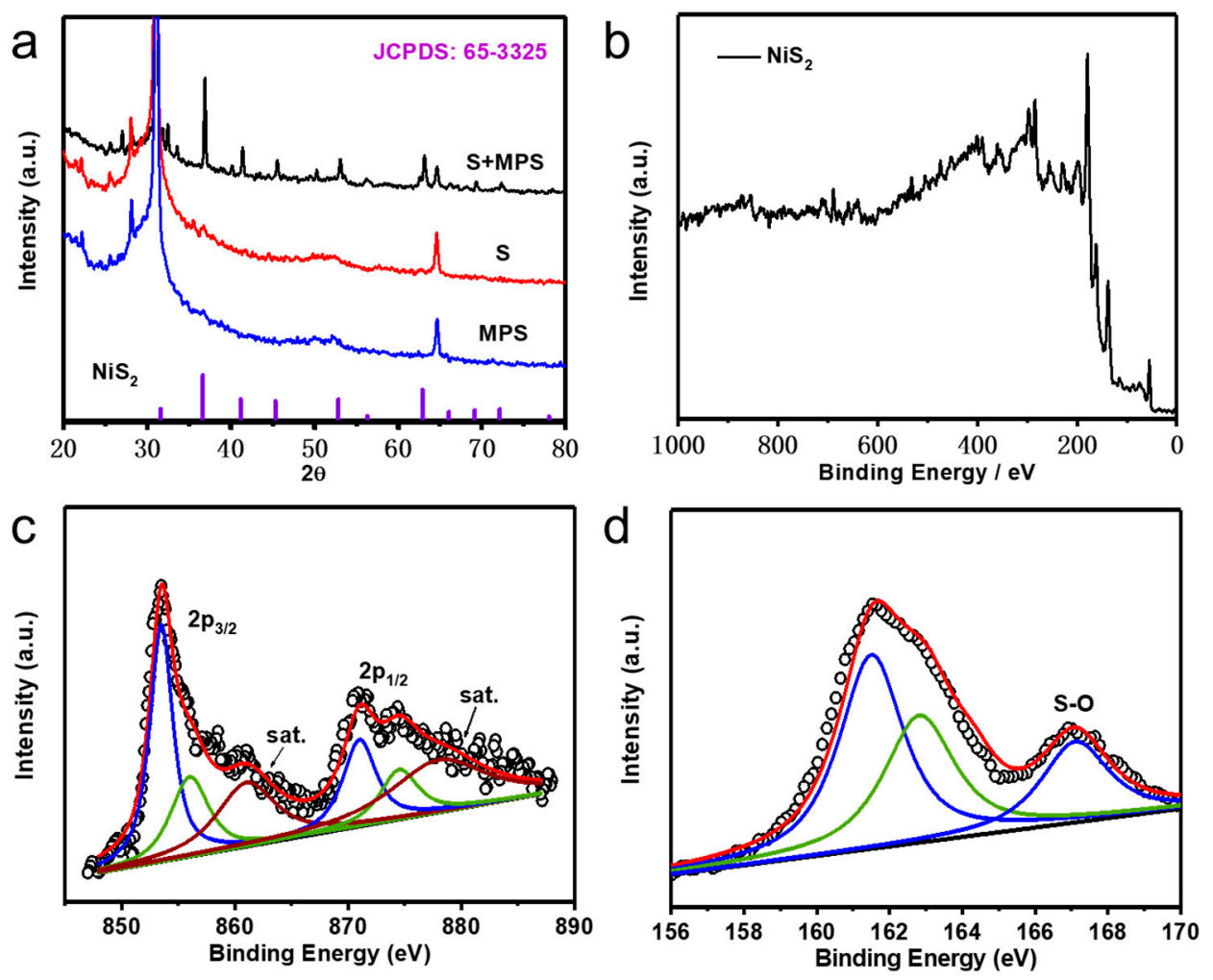

Figure 2. (a) XRD pattern of $\mathrm{NiS}_{2}$ prepared from three different sulfur sources; (b) X-ray photoelectron spectroscopy (XPS) survey spectra of $\mathrm{NiS}_{2}$; High-resolution XPS spectra of (c) Ni 2p, (d) S 2p of samples.

In order to further prove the applicability of the synthesized $\mathrm{NiS}_{2}$, electrochemical analysis was carried out to investigate the HER performance of the electrocatalyst in $0.5 \mathrm{M} \mathrm{H}_{2} \mathrm{SO}_{4}$ aqueous solution. Linear scan voltammetry (LSV) curves were measured to analyze the HER activity of $\mathrm{NiS}_{2}$, as shown in Figure 3a. The electrode based on $\mathrm{NiS}_{2}$ and grown on carbon paper from MPS and sulfur powder showed excellent HER performance, with an overpotential of $131 \mathrm{mV}$ to drive $-10 \mathrm{~mA}$ $\mathrm{cm}^{-1}$. Compared with the performance of the $\mathrm{NiS}_{2}$ synthesized using MPS or sulfur powder as the sulfur source, the overpotential was $197 \mathrm{mV}$ and $261 \mathrm{mV}$, respectively. There may be two reasons for switching on the voltage gap. First, the increase in the loading of the substrate was obvious; provided more active sites for hydrogen adsorption and desorption. From the SEM image, it can be seen that there were many substrates on the surface that is synthesized by the dual sulfur source, which may have caused more defects in the boundary crystal planes, thereby improving the catalytic performance to a certain extent. It can be seen from the XRD pattern that the crystallinity of the sample prepared 
using the dual sulfur source was better. Combined with the TEM image of the sample, the high crystal plane (200) of the sample can be seen. This sample showed excellent performance in the process of electrocatalytic water decomposition. We summarized the overpotential of the HER of similar samples that have been published so far; Figure S3. Figure $3 b$ shows the Tafel slope of $50 \mathrm{mV} \mathrm{dec}^{-1}$ for $\mathrm{NiS}_{2}$ with MPS and sulfur powder and the other two Tafel slopes of $53 \mathrm{mV} \mathrm{dec}^{-1}$ and $74 \mathrm{mV} \mathrm{dec}^{-1}$ for $\mathrm{NiS}_{2}$ with MPS or sulfur powder, respectively. The lowest overpotential and Tafel slope for $\mathrm{NiS}_{2}$ fabricated by MPS and sulfur powder indicated the best performance of hydrogen evolution reaction and high intrinsic catalytic activity supported by its superior exchange current density. To assess the stability of the catalytic performance of $\mathrm{NiS}_{2}$, cycling voltammetry (CV) was conducted. Thus, after 1000 cycles of stability testing at $-10 \mathrm{~mA} \mathrm{~cm}^{-1}$, the performance loss was only $5 \mathrm{mV}$.
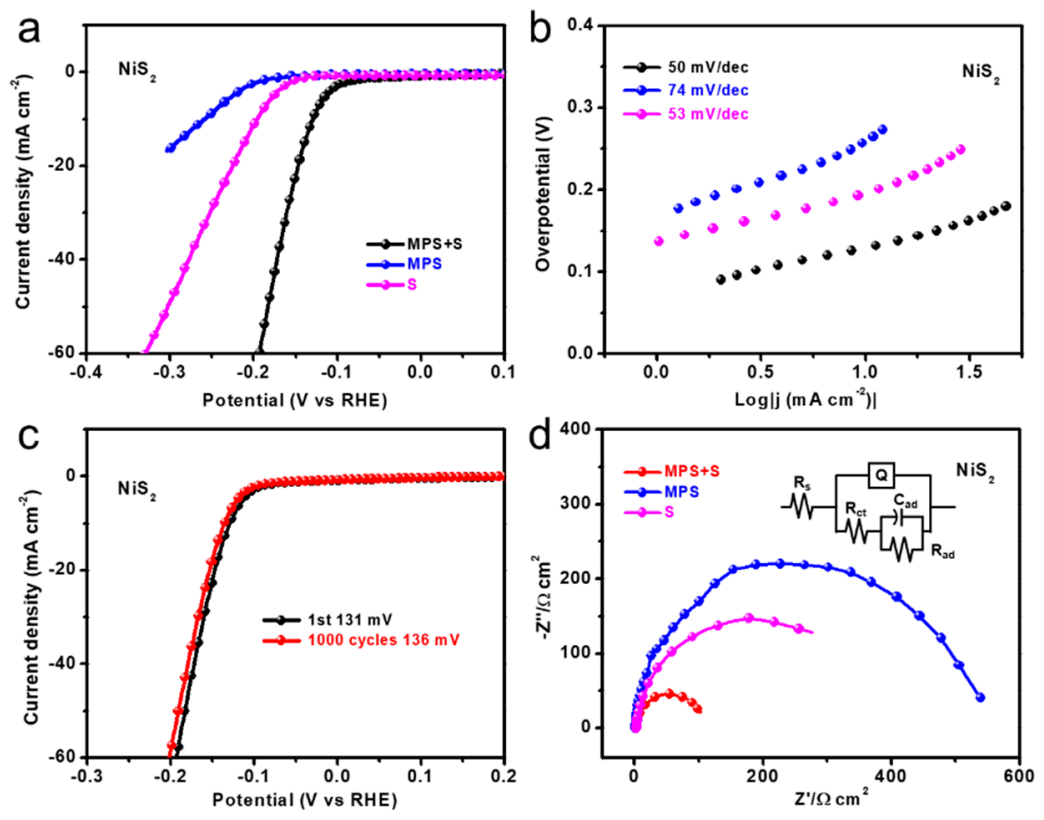

Figure 3. (a) LSV curves of $\mathrm{NiS}_{2}$ fabricated from different sulfur sources. (b) The corresponding Tafel plots of samples; (c) LSV curves before and after 1000 CV cycles and (d) electrochemical impedance spectroscopy of samples.

Electrochemical impendence spectroscopy (EIS) was used to assess the charge transfer performance of the synthesized $\mathrm{NiS}_{2}$, which also determined the double layer capacitance [1,16]. The Nyquist plots (Figure 3d) confirmed that the $\mathrm{NiS}_{2}$ fabricated from MPS and sulfur powder showed excellently smaller charge transfer resistance $\left(\mathrm{R}_{c t}\right)$, compared to other two materials, in the experiment and simulation in $0.5 \mathrm{M} \mathrm{H}_{2} \mathrm{SO}_{4}$ solution. The experimental data was fitted according to the electrical model presented in the inset of Figure $3 \mathrm{~d} . \mathrm{C}_{a d}$ and $\mathrm{R}_{a d}$ represent adsorption capacitance and adsorption resistance, respectively. The lowest charge transfer resistance of $53 \Omega$ for $\mathrm{NiS}_{2}$ prepared by MPS and sulfur powder was observed from the fitting experiment data in Figure $3 \mathrm{~d}$. The charge transfer resistances of $\mathrm{NiS}_{2}$ with only MPS and only sulfur powder were $178 \Omega$ and $258 \Omega$, respectively. Contrasting the value of $\mathrm{R}_{c t}$ of the samples, it can be observed that the $\mathrm{NiS}_{2}$ fabricated by MPS and sulfur powder had improved electrical conductivity, allowing electrochemical processes to occur on the surface. Nonfaradaic double-layer capacitance $\left(C_{d l}\right)$ was calculated for further analysis of the $\mathrm{NiS}_{2}$ activity. The $\mathrm{C}_{d l}$ of $\mathrm{NiS}_{2}$ fabricated by the dual sulfur source was $10.3 \mathrm{mF} \mathrm{cm}{ }^{-2}$, and the $\mathrm{C}_{d l}$ of the material prepared by MPS and sulfur powder were $0.44 \mathrm{mF} \mathrm{cm}^{-2}$ and $1.67 \mathrm{mF} \mathrm{cm}^{-2}$, respectively. Different scan rates of cyclic voltammetry measurement were carried out in order to determine the electrochemical surface area (ECSA) of $\mathrm{NiS}_{2}$, as shown in Figure $4 \mathrm{a}$. The catalytic performance of the working electrode was normalized to $1 \mathrm{~cm}^{2}$. Figure $4 \mathrm{a}-\mathrm{d}$ show cyclic voltammograms of $\mathrm{NiS}_{2}$ prepared by different 
experimental schemes which were measured in the nonfaradaic capacitance current range with different scan rates.
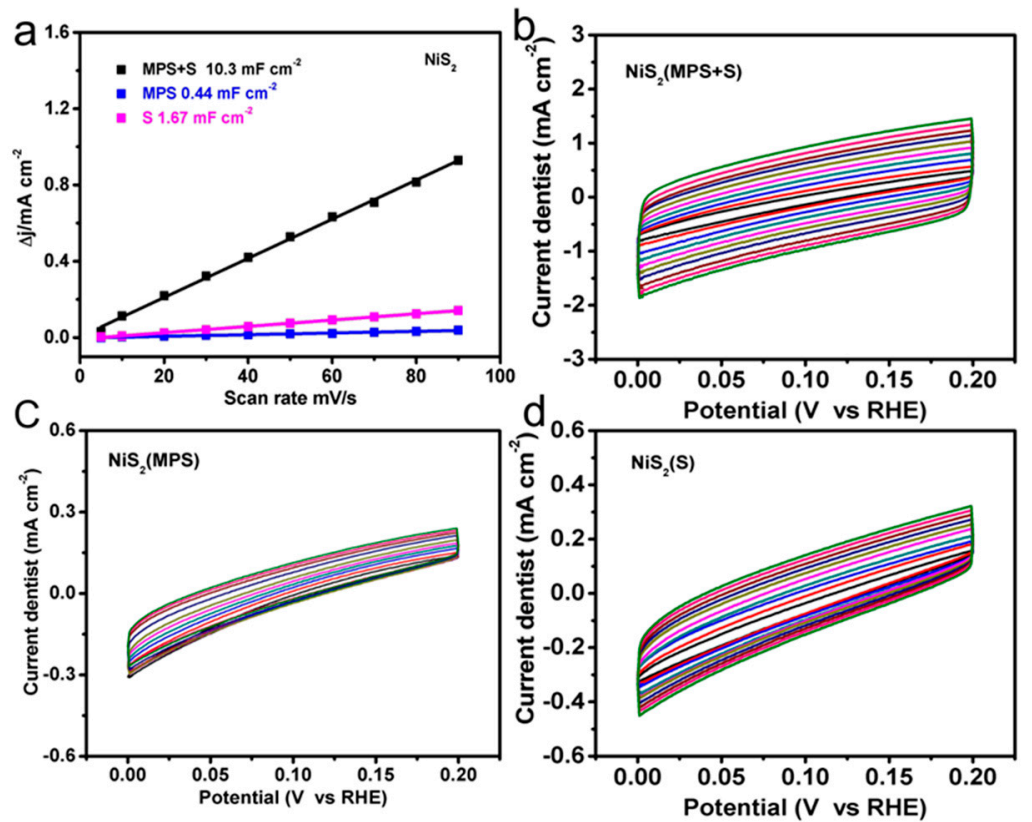

Figure 4. (a) The double-layer capacitance $\left(C_{d l}\right)$ calculated by liner fitting of the capacitive currents of different catalysts versus scan rate. (b-d) Cyclic voltammograms of $\mathrm{NiS}_{2}$ prepared by different experimental schemes.

\section{Conclusions}

In this work, we proposed a one-step hydrothermal method for $\mathrm{NiS}_{2}$ (MS) synthesis from dual sulfur sources and applied the obtained material as a catalyst for HER. The resulting material had a regular morphology with more specific surface area and active sites. The electrochemical performance and stability of $\mathrm{NiS}_{2}$ prepared by the hydrothermal method improved significantly. We believe that our findings will have a positive impact on the industrial application of nonprecious metal catalysts.

Supplementary Materials: The following are available online at http://www.mdpi.com/2079-4991/10/11/2115/s1, Figure S1. (a) and (b) are SEM images of $\mathrm{NiS}_{2}$ fabricated by MPS as the only sulfur source. Figure S2. (a) and (b) are SEM patterns of the product which prepared by sulfur powder only. Figure S3. Contrast of HER activity of electrocatalysts of similar materials.

Author Contributions: Conceptualization, S.Y. and K.W.; methodology, K.W., H.S. and Y.G.; validation, Y.G., Z.P. and K.W.; investigation, K.W. and Y.G.; data curation, K.W. and Y.G.; writing-original draft preparation, K.W. and Y.G.; writing-review and editing, S.Y.; supervision, K.W.; project administration, S.Y.; funding acquisition, S.Y.; Z.L. contribution is TEM test; X.D. contribution is XRD test. All authors have read and agreed to the published version of the manuscript.

Funding: This work is supported by the National Science Foundations of China (No. 61991431, No. 11574136), the National Basic Research Program of China (2018YFA0209101).

Conflicts of Interest: The authors declare no competing financial interest.

\section{References}

1. Tang, C.; Pu, Z.; Liu, Q.; Asiri, A.M.; Jiang, P. NiS 2 nanosheets array grown on carbon cloth as an efficient 3D hydrogen evolution cathode. Electrochim. Acta 2015, 153, 508-514. [CrossRef]

2. Yu, L.; Zhou, H.; Sun, J.; Qin, F.; Yu, F.; Bao, J.; Yu, Y.; Chen, S.; Ren, Z. Cu nanowires shelled with NiFe layered double hydroxide nanosheets as bifunctional electrocatalysts for overall water splitting. Energy Environ. Sci. 2017, 10, 1820-1827. [CrossRef] 
3. Seh, Z.W.; Kibsgaard, J.; Dickens, C.F.; Chorkendorff, I.; Nørskov, J.K.; Jaramillo, T.F. Combining theory and experiment in electrocatalysis: Insights into materials design. Science 2017, 355, eaad4998. [CrossRef] [PubMed]

4. Zhou, H.; Yu, F.; Huang, Y.; Sun, J.; Zhu, Z.; Nielsen, R.J.; He, R.; Bao, J.; Iii, W.A.G.; Chen, S.; et al. Efficient hydrogen evolution by ternary molybdenum sulfoselenide particles on self-standing porous nickel diselenide foam. Nat. Commun. 2016, 7, 12765. [CrossRef] [PubMed]

5. Tang, C.; Zhang, R.; Lu, W.; Wang, Z.; Liu, D.; Hao, S.; Du, G.; Asiri, A.M.; Sun, X. Energy-Saving Electrolytic Hydrogen Generation: $\mathrm{Ni}_{2} \mathrm{P}$ Nanoarray as a High-Performance Non-Noble-Metal Electrocatalyst. Angew. Chem. Int. Ed. 2016, 56, 842-846. [CrossRef] [PubMed]

6. Li, X.; Hao, X.; Abudula, A.; Guan, G. Nanostructured catalysts for electrochemical water splitting: Current state and prospects. J. Mater. Chem. A 2016, 4, 11973-12000. [CrossRef]

7. $\mathrm{Hu}, \mathrm{C}$;; Chen, X.; Dai, Q.; Wang, M.; Qu, L.; Dai, L. Earth-abundant carbon catalysts for renewable generation of clean energy from sunlight and water. Nano Energy 2017, 41, 367-376. [CrossRef]

8. Ying, Z.; Zheng, X.; Cui, G. Detailed kinetic study of the electrochemical Bunsen reaction in the sulfur-iodine cycle for hydrogen production. Energy Convers. Manag. 2016, 115, 26-31. [CrossRef]

9. Hu, S.; Xu, L.; Wang, L.; Li, D.; Zhang, P.; Chen, S. Activity and stability of monometallic and bimetallic catalysts for high-temperature catalytic $\mathrm{HI}$ decomposition in the iodine-sulfur hydrogen production cycle. Int. J. Hydrogen Energy 2016, 41, 773-783. [CrossRef]

10. Wu, A.; Xie, Y.; Ma, H.; Tian, C.; Gu, Y.; Yan, H.; Zhang, X.; Yang, G.; Fu, H. Integrating the active OER and HER components as the heterostructures for the efficient overall water splitting. Nano Energy 2018, 44, 353-363. [CrossRef]

11. Wang, Y.; Kong, B.; Zhao, D.; Wang, H.; Plebanski, M. Strategies for developing transition metal phosphides as heterogeneous electrocatalysts for water splitting. Nano Today 2017, 15, 26-55. [CrossRef]

12. Duan, J.; Chen, S.; Zhao, C. Ultrathin metal-organic framework array for efficient electrocatalytic water splitting. Nat. Commun. 2017, 8, 15341. [CrossRef]

13. Kumar, A.; Bhattacharyya, S. Porous NiFe-Oxide Nanocubes as Bifunctional Electrocatalysts for Efficient Water-Splitting. ACS Appl. Mater. Interfaces 2017, 9, 41906-41915. [CrossRef]

14. Tian, J.; Liu, Q.; Asiri, A.M.; Sun, X. Self-Supported Nanoporous Cobalt Phosphide Nanowire Arrays: An Efficient 3D Hydrogen-Evolving Cathode over the Wide Range of $\mathrm{pH} 0-14$. J. Am. Chem. Soc. 2014, 136, 7587-7590. [CrossRef]

15. Hu, J.; Zhang, C.; Yang, S.; Lin, H.; Hu, C.; Long, X.; Yang, S. Hydrogen evolution electrocatalysis with binary-nonmetal transition metal compounds. J. Mater. Chem. A 2017, 5, 5995-6012. [CrossRef]

16. Faber, M.S.; Lukowski, M.A.; Ding, Q.; Kaiser, N.S.; Jin, S. Earth-Abundant Metal Pyrites $\left(\mathrm{FeS}_{2}, \mathrm{CoS}_{2}, \mathrm{NiS}_{2}\right.$, and Their Alloys) for Highly Efficient Hydrogen Evolution and Polysulfide Reduction Electrocatalysis. J. Phys. Chem. C 2014, 118, 21347-21356. [CrossRef]

17. An, T.; Wang, Y.; Tang, J.; Wei, W.; Cui, X.; Alenizi, A.M.; Zhang, L.; Zheng, G. Interlaced $\mathrm{NiS}_{2}-\mathrm{MoS}_{2}$ nanoflake-nanowires as efficient hydrogen evolution electrocatalysts in basic solutions. J. Mater. Chem. A 2016, 4, 13439-13443. [CrossRef]

18. Yin, J.; Li, Y.X.; Lv, F.; Lu, M.; Sun, K.; Wang, W. Oxygen vacancies dominated $\mathrm{NiS}_{2} / \mathrm{CoS}_{2}$ interface porous nanowires for portable $\mathrm{Zn}$-air batteries driven water splitting devices. Adv. Mater. 2017, 29, 1704681. [CrossRef] [PubMed]

19. Tian, T.; Huang, L.; Ai, L.; Jiang, J. Surface anion-rich $\mathrm{NiS}_{2}$ hollow microspheres derived from metal-organic frameworks as a robust electrocatalyst for the hydrogen evolution reaction. J. Mater. Chem. A 2017, 5, 20985-20992. [CrossRef]

20. Wu, X.; Yang, B.; Li, Z.; Lei, L.; Zhang, X. Synthesis of supported vertical $\mathrm{NiS}_{2}$ nanosheets for hydrogen evolution reaction in acidic and alkaline solution. RSC Adv. 2015, 5, 32976-32982. [CrossRef]

21. Lin, J.H.; Wang, P.C.; Wang, H.H.; Li, C. Defect-Rich Heterogeneous $\mathrm{MoS}_{2} / \mathrm{NiS}_{2}$ Nanosheets Electrocatalysts for Efficient Overall Water Splitting. Adv. Sci. 2019, 6, 1900246. [CrossRef] [PubMed]

22. Liu, H.; He, Q.; Jiang, H.; Lin, Y.; Zhang, Y.; Habib, M.; Chen, S.; Song, L. Electronic Structure Reconfiguration toward Pyrite $\mathrm{NiS}_{2}$ via Engineered Heteroatom Defect Boosting Overall Water Splitting. ACS Nano 2017, 11, 11574-11583. [CrossRef]

23. Xu, S.; Jiang, W.-J.; Lian, Y.; Li, H.; Hong, S.; Xu, S.; Yan, H.; Hu, J.-S. NiS 2 nanodotted carnation-like CoS ${ }_{2}$ for enhanced electrocatalytic water splitting. Chem. Commun. 2019, 55, 3781-3784. [CrossRef] 
24. Hao, J.; Yang, W.; Hou, J.; Mao, B.; Huang, Z.; Shi, W. Nitrogen doped $\mathrm{NiS}_{2}$ nanoarrays with enhanced electrocatalytic activity for water oxidation. J. Mater. Chem. A 2017, 5, 17811-17816. [CrossRef]

25. Huang, T.; Fang, H.; Xu, L.; Wang, Z.; Xin, Y.; Yu, J.; Yao, S.; Zhang, Z. Electrocatalytic performance of cubic $\mathrm{NiS}_{2}$ and hexagonal NiS for oxygen reduction reaction. J. Catal. 2018, 359, 223-232. [CrossRef]

26. Kuang, P.Y.; He, M.; Zhu, B.C.; Yu, J.G.; Fan, K. OD/2D NiS $2 /$ V-MXene composite for electrocatalytic $\mathrm{H}_{2}$ evolution. J. Catal. 2019, 375, 8-20. [CrossRef]

27. Liu, P.; Li, J.; Lu, Y.; Xiang, B. Facile synthesis of $\mathrm{NiS}_{2}$ nanowires and its efficient electrocatalytic performance for hydrogen evolution reaction. Int. J. Hydrogen Energy 2018, 43, 72-77. [CrossRef]

28. Wei, W.; Mi, L.; Gao, Y.; Zheng, Z.; Chen, W.; Guan, X. Partial Ion-Exchange of Nickel-Sulfide-Derived Electrodes for High Performance Supercapacitors. Chem. Mater. 2014, 26, 3418-3426. [CrossRef]

29. Yu, B.; Wang, X.; Qi, F.; Zheng, B.; He, J.; Lin, J.; Zhang, W.; Li, Y.; Chen, Y. Self-Assembled Coral-like Hierarchical Architecture Constructed by $\mathrm{NiSe}_{2}$ Nanocrystals with Comparable Hydrogen-Evolution Performance of Precious Platinum Catalyst. ACS Appl. Mater. Interfaces 2017, 9, 7154-7159. [CrossRef]

30. Liu, Z.; Zheng, X.; Luo, S.-L.; Xu, S.-Q.; Yuan, N.-Y.; Ding, J.-N. High performance Li-S battery based on amorphous $\mathrm{NiS}_{2}$ as the host material for the $\mathrm{S}$ cathode. J. Mater. Chem. A 2016, 4, 13395-13399. [CrossRef]

31. Zhang, X.-J.; Wang, S.-W.; Wang, G.-S.; Li, Z.; Guo, A.-P.; Zhu, J.-Q.; Liu, D.-P.; Yin, P.-G. Facile synthesis of $\mathrm{NiS}_{2} @ \mathrm{MoS}_{2}$ core-shell nanospheres for effective enhancement in microwave absorption. RSC Adv. 2017, 7, 22454-22460. [CrossRef]

Publisher's Note: MDPI stays neutral with regard to jurisdictional claims in published maps and institutional affiliations. 